\title{
POTENCIAL EVOCADO SOMATO-SENSITIVO EM CRIANÇAS COM PONTAS EVOCADAS POR ESTÍMULO SOMATO- SENSITIVO NO ELETRENCEFALOGRAMA
}

\author{
Gloria M.A.S. Tedrus' ${ }^{1}$ Lineu Corrêa Fonseca ${ }^{1}$
}

\begin{abstract}
RESUMO - Estudamos as características dos componentes corticais do potencial evocado somato-sensitivo (PES) de 31 crianças com pontas evocadas ao EEG (PE), das quais 20 com epilepsias benignas da infância (EBIPE) e 11 crianças sem epilepsia (PENE). Os dados foram comparados com um grupo de 20 "crianças normais". A amplitude do N60 e $N 75$ foi maior nos grupos EBIPE e PENE em relação ao grupo normal. 0 P98 mostrou amplitude significantemente maior no grupo PENE do que no grupo normal. 0 achado de PES de amplitudes elevadas (N75 e P98) foi maior no grupo de crianças com PE do que no grupo normal. 0 exato mecanismo envolvido na gênese da PE e de componentes corticais com amplitudes elevadas não está esclarecido. Um provável mecanismo seria a hiperexcitabilidade cortical focal, de natureza funcional, não lesional. Não foi observada diferença nos componentes dos PES nas crianças com PE segundo a ocorrência ou não de epilepsia.
\end{abstract}

PALAVRAS-CHAVE: potencial evocado somato-sensitivo, eletrencefalograma, ponta evocada.

\begin{abstract}
Somatosensory evoked potential in children with evoked spikes by tapping of the feet or hands on electroencephalogram

ABSTRACT - Objective: To study the characteristics of Somatosensory evoked potential (SEP) cortical components in children with evoked spikes (ES) on EEG. Method: The children were aged 7-12 years, had normal neurological examination and neuropsychomotor development, and did not present signs/symptoms of CNS lesions. Data were compared within a group of 20 "normal" children. Results: The amplitude of the cortical components N75 and P98, obtained by posterior tibial nerve stimulation, was higher in ES groups as compared with the normal group. Conclusion: High amplitude SEP (N75 and P98) has higher values in the group of children with ES than in the normal group. The exact mechanism involved in the genesis of ES and high amplitude SEP is not clear yet. A possible mechanism would be focal cortical hyperexcitability related to functional activity. No difference was observed in SEP components in children with ES considering occurrence or not of epilepsy. Therefore, SEP does not bring elements to distinguish between the groups suffering or not epilepsy.
\end{abstract}

KEY WORDS: somatosensory evoked potential, electroencephalogram, evoked spike.

Pontas evocadas, no eletrencefalograma (EEG), podem ser obtidas pela percussão dos pés e mãos ( $\mathrm{PE}$ ) ou por estímulos elétricos ou mecânicos. Mais comumente tem sido aplicado como estímulo a percussão com martelo de reflexos com intensidade similar à utilizada na pesquisa dos reflexos profundos ${ }^{1,2}$. As porções distais dos membros inferiores (calcanhar ou artelhos) ou membros superiores (dedos ou região dorsal da mão) parecem ser os locais mais apropriados para a estimulação e obtenção de PE. A cada estímulo corresponde uma ponta bifásica ou polifásica, por vezes seguida de onda lenta. PE foram registradas em 1 a $3 \%$ das crianças examinadas em serviços de eletrencefalografia ${ }^{2-4}$. As PE decorrem de hiperexcitabilidade cortical, que também seria responsável pelo aparecimento de pontas espontâneas no EEG e poderia originar crises epilépticas. Na maioria dos casos, PE são registradas em crianças sem evidências de lesão cerebral, 0 que sugere mecanismo funcional, não lesional, ligado à idade e possivelmente genético na sua gênese. Atividade epileptiforme espontânea pode ocorrer no EEG de crianças com PE e, neste caso, há maior associação com crises epilépticas ${ }^{1,2}$. PE são encontradas mais comumente em crianças sem crises epilépticas, e, entre as crianças com epilepsia, predominantemente naquelas com epilepsias parciais idiopáticas (EPI). Nestas epilepsias a evolução é favorável e não há lesão cerebral. Podem ocorrer PE, também, em epilepsias generalizadas idiopáticas ou criptogênicas e parciais sintomáticas $s^{3-8}$.

0 potencial evocado somato-sensitivo (PES) tem sido estudado desde 1950 em pacientes adultos com patologias

1Professor Titular de Neurologia, Faculdade de Medicina Pontifícia Universidade Católica de Campinas, Campinas SP, Brasil.

Recebido 4 Setembro 2003, Aceito 15 Dezembro 2003.

Dra. Gloria M.A.S. Tedrus - Rua Sebastião de Souza 205/122 - 13020-020 Campinas SP - Brasil. E-mail: tedrusbento@uol.com.br 
neurológicas mas só nos últimos anos passou a ser valorizado como rotina diagnostica em neurologia infantil ${ }^{9-12}$. Em estudos do PES nas epilepsias parciais idiopáticas da infância foi observado que os componentes corticais precoces apresentam latências e amplitudes normais, enquanto os de média e longa latência podem apresentar amplitudes gigantes, mas morfologia e latências normais ${ }^{8,13-17}$. Não encontramos na literatura estudo específico de PES em crianças com PE e sem crises epilépticas, o que poderia contribuir para melhor compreensão dos mecanismos envolvidos na gênese das PE. 0 objetivo deste trabalho é estudar a latência e a amplitude dos componentes corticais dos PES em crianças com PE no EEG levando em conta os aspectos clínicos e a ocorrência de atividade epileptiforme, sua lateralização e localização.

\section{MÉTODO}

No período de 1997 a 2000, foram selecionadas 31 crianças atendidas nos ambulatórios de Neurologia infantil do Hospital e Maternidade Celso Pierro da Pontifícia Universidade Católica de Campinas (HMCP-PUC-Campinas). As crianças tinham de 7 a 12 anos de idade, com exame neurológico e desenvolvimento neuropsicomotor normais, ausência de dados de anamnese ou de exames laboratoriais sugestivos de lesão do sistema nervoso central (SNC), e apresentavam, no EEG, pontas evocadas pela percussão dos pés e/ou mãos (Fig 1) e atividade de base normal. As crianças foram classificadas em 2 grupos, um deles de 20 casos que apresentavam epilepsias benignas da infância (EBIPE) e outro com 11, sem crises epilépticas que apresentavam cefaléia e distúrbio de aprendizado ou de comportamento (PENE).

Foi realizada anamnese específica, exame clínico-neurológico, EEG e o PES, com um período máximo de 30 dias, entre eles.

Os EEG foram obtidos no serviço de eletrencefalografia do HM CP. PUC-Campinas. Foi utilizado o sistema internacional 10-20 de colocação de eletrodos ${ }^{18}$. 0 exame foi obtido durante vigília e, quando possível, durante sono espontâneo. Foi feita a percussão da região plantar dos pés, dorso das mãos, dedos e artelhos, com técnica semelhante à usada na pesquisa de reflexos profundos, percutindo-se cinco vezes o local. Foram analisados, visualmente ao EEG, os seguintes elementos: atividade elétrica cerebral de base; atividade epileptiforme quanto à localização, lateralização, morfologia e amplitude; PE quanto ao local dos estímulos que as evocaram, assim como sua localização, morfologia e amplitude.

Os PES foram realizados no serviço de Potencial Evocado do Hospital de Clínicas da UNICAMP, sendo utilizado aparelho NihonKoden modelo Neuropack Four. 0 exame foi realizado com a criança acordada, em decúbito dorsal, em ambiente escurecido, mantendose a temperatura ambiente constante. Utilizou-se o sistema 10-20 de colagem de eletrodos. Os eletrodos foram colocados no ponto de Erb, Fz e nas posições $C^{\prime} 4, C^{\prime} 3$ e C'z, respectivamente, $2 \mathrm{~cm}$ atrás da posição C4, C3 e Cz .

Foram estimulados os nervos mediano e tibial posterior, em ambos os membros, separadamente. A estimulação no nervo mediano foi feita acima da prega do punho, e a estimulação do nervo tibial posterior foi aplicada próximo ao maléolo interno.

A impedancia dos eletrodos foi mantida abaixo de 5 Kohms. 0 impulso elétrico foi constituído por um pulso quadrado de corrente contínua com freqüência de $2 \mathrm{~Hz}$ e duração de 0,2 ms. Foi utilizado a intensidade de estimulação logo acima da que provocou resposta motora do quinto dedo ou artelho. $A$ análise de tempo utilizada foi $100 \mathrm{~ms}$ ou $500 \mathrm{~ms}$. A sensibilidade foi ajustada em $10 \mu \mathrm{Volts} / \mathrm{cm}$ e os filtros regulados em $1 \mathrm{~Hz}$ e $3000 \mathrm{~Hz}$. Foi feita promediação de 500 respostas. Foram utilizadas as derivações C'z - Fz quando da estimulação do nervo tibial posterior e $C^{\prime}$ contralateral - $C^{\prime}$ ipsilateral para a estimulação do nervo mediano.

Foram medidas as latências, em ms, e as amplitudes em $\mu \mathrm{V}$, dos componentes N9, N20, P25, N35, P45, N60 e P100 obtidos pela estimulação dos nervos medianos esquerdo e direito e P37, N45, P60, N75, P98 e N126 obtidos pela estimulação dos nervos tibiais posteriores esquerdo e direito.

Foram estudadas as latências e as amplitudes dos componentes corticais dos PES.

Os dados obtidos foram comparados com os de um grupo controle de 20 crianças "normais", de mesma faixa etária, sem antecedentes sugestivos de patologias com envolvimento do SNC, exame neurológico e EEG normais.

Nas comparações em que foram evidenciadas diferenças significativas de amplitude entre os grupos, foi estabelecida como "amplitude elevada" de um determinado componente aquela que melhor discriminasse os grupos. Foi, então, comparada a ocorrência de componentes de amplitude elevada nos vários grupos. Foi comparada a ocorrência de um ou mais dos componentes corticais com amplitude elevada nos indivíduos dos grupos.

Para comparação das variáveis contínuas entre os grupos utilizouse 0 teste de Wilcoxon para amostras independentes (Mann-Whitney). Na comparação de proporções para variáveis categóricas entre os grupos utilizou-se o teste Qui-quadrado e o teste Exato de Fisher quando os critérios de aplicação do Qui-quadrado não foram atendidas. Para a rejeição da hipótese de nulidade foi estabelecido para todos os testes, um risco $\alpha<0,05$ ou $5 \%$.

A inclusão no estudo somente ocorreu após o consentimento pelos responsáveis e com a aprovação pelo Comitê de Ética em Pesquisa

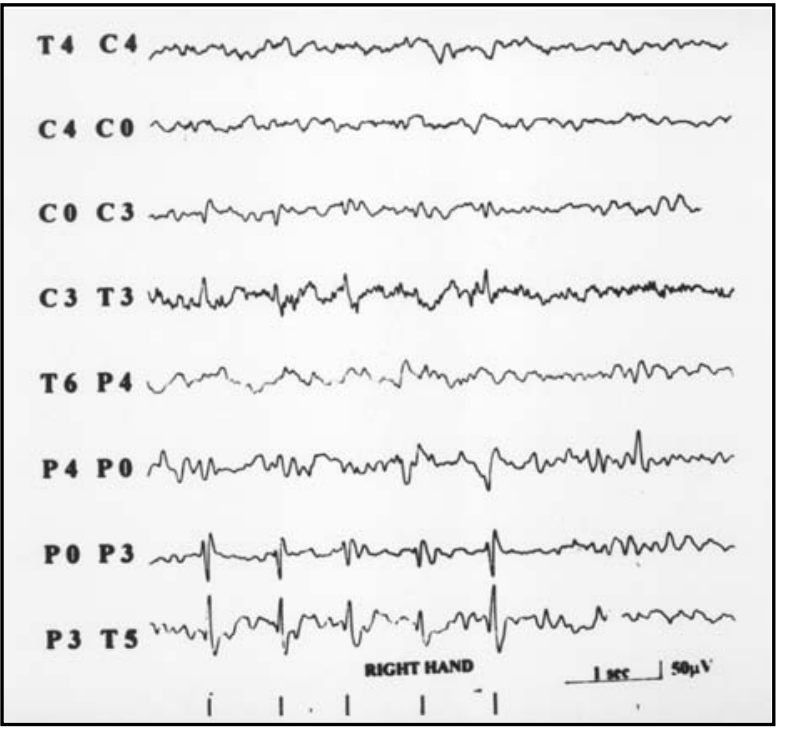

Fig 1. Exemplo de EEG com pontas nas regiões central e parietal esquerdas, evocadas pela percussão da mão direita. 
da PUC-Campinas.

\section{RESULTADOS}

Componentes corticais do PES pela estimulação dos nervos mediano e tibial posterior foram obtidos em todas as crianças estudadas.

0 estudo das latências dos componentes corticais do PES obtidos pela estimulação dos nervos medianos e tibiais posteriores não revelou diferenças significativas nas comparações entre os grupos PENE, EBIPE e normal.

Amplitude dos componentes corticais do PES obtidos pela estimulação do nervo mediano

0 componente $\mathrm{N} 60 \mathrm{com}$ amplitude elevada (maior que 7 $\mu \mathrm{V})$ foi significativamente mais freqüente nos grupos PENE $e$ EBIPE do que no grupo normal (Teste exato de Fisher, $p<0,01$ ). Não houve diferença significativa entre os grupos PENE e EBIPE (Tabela 1).

Para os demais componentes não houve diferenças significativas entre os grupos PENE, EBIPE e normal.

Amplitude dos componentes corticais do PES obtidos pela estimulação do nervo tibial posterior

Componentes N45, P98 e N126 com amplitudes elevadas (maior que $10 \mu \mathrm{V}$ ) foram observados em proporção significantemente maior das crianças do grupo EBIPE do que dos grupos PENE e normal. Os componentes N75 com amplitudes elevadas (maior que $10 \mu \mathrm{V}$ ) estavam presentes de modo significantemente maior dos indivíduos dos grupos PENE e EBIPE do que do grupo normal. Não houve diferença significativa entre os grupos PENE e EBIPE (Tabela 1).

A amplitude do componente cortical $\mathrm{N} 75$ pela estimulação à direita foi maior nos grupos PENE e EBIPE em relação ao grupo normal mas com tendência estatística para PE-NE x normal (teste de Mann-Whitney, $p=0,06$ ) e de modo significativo para EBIPE $x$ normal (teste de Mann-Whitney, $p=0,046$ ).

A amplitude do componente P98 mostrou, à direita, amplitude significantemente maior no grupo PENE do que no grupo normal (teste de $M$ ann-Whitney, $p=0,005$ ) e à esquerda a amplitude também foi maior mas com tendência a significancia estatística (teste de $M$ ann-Whitney, $p=0,06$ ). Não houve diferença significativa, para o P98, entre os grupos PENE e EBIPE. Neste último grupo a amplitude também foi maior que no grupo normal, para estimulação à esquerda ( $p=0,01$ ) (Tabela 2) (Fig.2).

\section{DISCUSSÃO}

Em acordo com o descrito na literatura, obtiveram-se os componentes corticais do PES em todas as crianças desta pesquisa, tanto pela estimulação dos nervos medianos e tibiais posteriores. Neste trabalho, não foi observada alteração nas latências dos componentes corticais dos PES nas crianças com e sem crises epilépticas e também com o grupo de crianças normais. Esse dado já havia sido referido em trabalho anterior quando comparamos grupos de crianças com epilepsias parciais idiopáticas e crianças normais ${ }^{19} \mathrm{e} \mathrm{também} \mathrm{por} \mathrm{outros} \mathrm{autores}{ }^{8,13-}$ 16,20. 0 aumento das latencias dos componentes corticais do PES foi referido em crianças com epilepsias sintomáticas ${ }^{8,21}$.

A amplitude elevada do componente $\mathrm{N} 60$ ( $\mathrm{n}$. mediano) foi mais freqüente nas crianças com crises epilépticas do que do grupo normal. Esse resultado está em acordo com os relatos da literatura ${ }^{8,15,16,22}$. A amplitude do componente P98 (n. tibial posterior) foi maior nas crianças do grupo PENE e EBIPE do que do grupo "normal" . Não se encontrou descrição análoga na literatura.

0 achado do componente $\mathrm{N} 75$ (n. tibial posterior) com amplitude elevada já havia sido relatado, anteriormente, nas epilepsias parciais idiopáticas da infância ${ }^{8,15,16,22}$. 0 significado neurofisiológico das pontas evocadas no EEG e dos componentes corticais com amplitudes elevadas do PES não está esclarecido. A observação das PE e dos componentes corticais com amplitude elevada, nas epilepsias parciais idiopáticas, sugere um mecanismo funcional, decorrente de hiperexcitabilidade cortical focal ou generalizada. Embora as PE possam estar presentes, também, em crianças com epilepsias sintomáticas, tais elementos são mais freqüentemente descritos nas epilepsias parciais idiopáticas, e são dependentes da idade ${ }^{2,48}$. A origem do componente cortical com amplitude elevada permanece especulativa, e o seu significado, a ser definido. Pode ser um indicador inespecífico de um evento neu-

Tabela 1. Componentes corticais N75 e P98 (nervo tibial posterior), membro estimulado, tamanho da amostra, médias de amplitudes e valores de p na comparações entre os grupos PENE, EBIPE e normal.

\begin{tabular}{|c|c|c|c|c|c|c|c|c|c|c|}
\hline \multirow[b]{2}{*}{$\begin{array}{l}\text { Componente } \\
\text { cortical }\end{array}$} & \multirow[b]{2}{*}{$\begin{array}{l}\text { Membro } \\
\text { estimulado }\end{array}$} & \multicolumn{3}{|c|}{$\begin{array}{l}\text { Tamanho } \\
\text { do grupo }\end{array}$} & \multicolumn{3}{|c|}{$\begin{array}{c}\text { Médias de } \\
\text { amplitude em }(\mu \mathrm{V})\end{array}$} & \multicolumn{3}{|c|}{$\begin{array}{c}\text { Valores } \\
\text { de P }\end{array}$} \\
\hline & & PENE & EBIPE & normal & PENE & EBIPE & normal & $\begin{array}{l}\text { PENE } x \\
\text { normal }\end{array}$ & $\begin{array}{l}\text { PENE X } \\
\text { EBIPE }\end{array}$ & $\begin{array}{l}\text { EBIPE } x \\
\text { normal }\end{array}$ \\
\hline \multirow[t]{2}{*}{ N75 } & Direito & 10 & 15 & 19 & 7,34 & 8,70 & 2,61 & 0,064 & 0,297 & $0,0467^{*}$ \\
\hline & Esquerdo & 9 & 13 & 17 & 7,76 & 5,55 & 3,28 & 0,161 & 0,30 & 0,572 \\
\hline \multirow[t]{2}{*}{ P98 } & Direito & 10 & 18 & 15 & 10,43 & 12,8 & 3,07 & $0,005^{*}$ & 0,486 & $0,080 *$ \\
\hline & Esquerdo & 8 & 17 & 16 & 8,20 & 10,34 & 2,65 & 0,060 & 0,662 & 0,018* \\
\hline
\end{tabular}




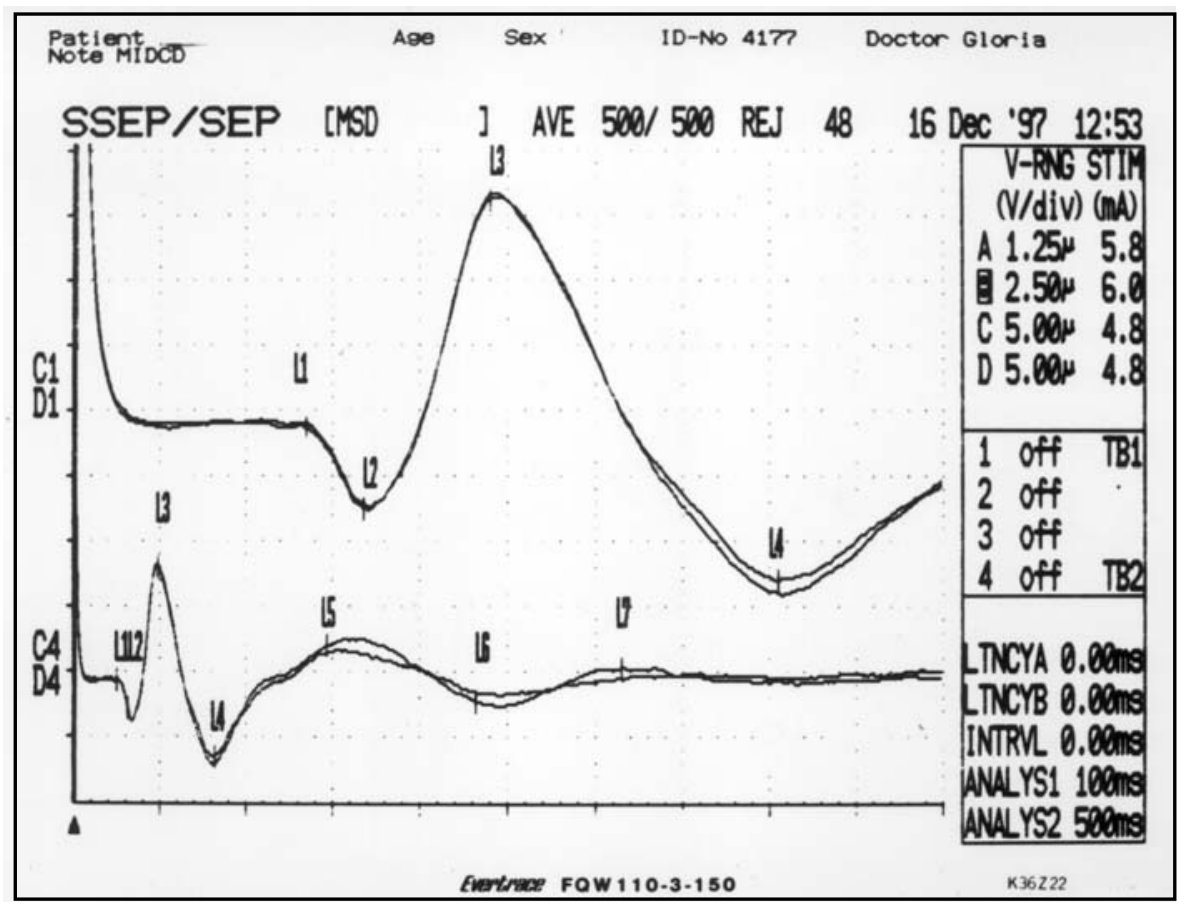

Fig 2. PES obtido pela estimulação do nervo tibial posterior direito, com componentes $N 75$ e P98 de amplitudes elevadas. A derivação utilizada foi $\mathrm{Cz}$ - Fz e com análise de tempo de 100 e $500 \mathrm{~ms}$ respectivamente, no C1, D1 e C4,D4.

Tabela 2. Distribuição das crianças nos grupos PENE, EBIPE e normal, segundo a ocorrência dos componentes N60 (n. mediano), N45, N75, P98 e $N 126$ (n. tibial posterior), com amplitudes elevadas ( > $7 \mu \mathrm{V}$ e $10 \mu \mathrm{V}$, respectivamente).

\begin{tabular}{|c|c|c|c|c|c|c|c|c|c|}
\hline \multirow[b]{3}{*}{$\begin{array}{l}\text { Componente } \\
\text { cortical }\end{array}$} & \multicolumn{6}{|c|}{ Ocorrência de componente com amplitude elevada } & & & \\
\hline & \multicolumn{3}{|c|}{ Presente } & \multicolumn{3}{|c|}{ Ausente } & \multicolumn{3}{|c|}{ Valores de P } \\
\hline & PENE & EBIPE & normal & PENE & EBIPE & normal & $\begin{array}{l}\text { PENE } x \\
\text { normal }\end{array}$ & $\begin{array}{c}\text { PENE X } \\
\text { EBIPE }\end{array}$ & $\begin{array}{l}\text { EBIPE x } \\
\text { normal }\end{array}$ \\
\hline N60 & 3 & 6 & 0 & 7 & 9 & 19 & $0,03 *$ & 0,46 & $0,01 *$ \\
\hline N45 & 1 & 7 & 1 & 8 & 11 & 19 & 0,53 & 0,14 & $0,01 *$ \\
\hline N75 & 3 & 5 & 0 & 7 & 11 & 20 & $0,029 *$ & 0,64 & $0,01 *$ \\
\hline P98 & 2 & 10 & 0 & 8 & 9 & 19 & 0,11 & 0,09 & $0,001 *$ \\
\hline N126 & 2 & 6 & 0 & 5 & 8 & 13 & 0,11 & 0,4 & $0,01 *$ \\
\hline
\end{tabular}

Teste exato de Fisher. * Diferenças estatisticamente significativas.

rológico e, em alguns pacientes, é indicativo de uma doença específica, como a epilepsia mioclônica progressiva ${ }^{23}$.

É bem conhecido que esses componentes com amplitude elevada são encontrados em pacientes com epilepsia mioclônica progressiva, mas, também ocorrem em outras afecções do SNC $C^{4,24-30}$. Esse aumento de amplitude dos componentes corticais do PES pode representar uma hiperexcitabilidade do córtex somato-sensitivo correspondente ao possível local epileptogênico ${ }^{29}$. Assim, esse componente poderia estar relacionado à predisposição a crises epilépticas, nas epilepsias parciais idiopáticas 1,4,13,16. Em apoio a esta idéia, há evidências de que as crianças com componentes corticais com amplitude elevada apresentam mais crises epilépticas do que a população geral $^{1,13}$. Tem sido sugerido por alguns autores, como possível patogênese do componente com amplitude elevada, um distúrbio dos interneurônios corticais inibidores ou uma falência nos mecanismos inibidores de estruturas subcorticais ${ }^{25,30}$.

0 significado neurofisiológico do componente cortical com amplitude elevada nas epilepsias parciais idiopáticas não está esclarecido. Não necessariamente existe uma relação 
entre a patogênese desses componentes com amplitude elevada nas epilepsias mioclônicas e nas parciais idiopáticas ${ }^{4}$. Em estudos evolutivos, Plasmati et al. ${ }^{15,16}$ observaram que 0 componente com amplitude elevada pode ser desencadeado pela estimulação de um membro ou lado e que pode haver mudança nessa localização durante a evolução, o que sugere um mecanismo funcional, não lesional, envolvido na gênese desse componente. Esses componentes, de média e longa latência, que se apresentam com amplitude elevada, podem resultar de uma hiperexcitabilidade cortical, seletivamente envolvendo as regiões parietal e parietal de associação, que são provavelmente as regiões onde estão localizados os geradores dos componentes N45, N75, P98 e N126. Outros autores 22,25 sugerem que diferentes mecanismos devem estar envolvidos na geração do componente cortical com amplitude elevada.

A evidência de que os componentes com amplitudes elevadas do PES, as PE e as pontas espontâneas no EEG possam apresentar geradores de mesma localização, e que esses geradores possam ser influenciados por estímulos aferentes, nos dá importantes informações dos mecanismos envolvidos na modulação da excitabilidade cortical na epilepsia parcial com pontas centrotemporais.

Neste trabalho houve diferenças significativas nos componentes dos PES na comparação entre as crianças sem epilepsia e com pontas evocadas por estímulos somato-sensitivos e as crianças normais, sugerindo uma excitabilidade cortical aumentada no grupo de estudo. Por outro lado, não foi evidenciada diferença nos componentes dos PES em crianças com pontas evocadas por estímulos somato-sensitivos segundo a ocorrência ou não de epilepsia. Os PES portanto não trazem elementos para a distinção entre os grupos com e sem epilepsia.

\section{REFERÊNCIAS}

1. DeMarco P. Evoked parietal spikes and childhood. Arch Neurol 1980;37:291-292.

2. Fonseca LC, Tedrus GMA. Pontas evocadas por estímulos somatossensitivos e síndromes epilépticas. In Costa JC, Palmini A, Yacubian EMT, Cavalheiro EA (eds). Fundamentos neurobiológicos das epilepsias: aspectos clínicos e cirúrgicos. São Paulo: Lemos Editorial, Gráficos Ltda. 1998:243-249.

3. DeMarco P, Tassinari CA. Extreme somatosensory evoked potential (ESEP): an EEG sign forecasting the possible occurence of seizures in children. Epilepsia 1981;22:569-575.

4. Micheloyannis I, Samara C, Liakakos T. Giant somatosensory evoked potentials in children without myoclonic epilepsy. Acta Neurol Scand 1989;79:146-149.

5. Negrin P, DeMarco P. Parietal focal spikes evoked by tactile somatotopic stimulation in sixty non-epileptic children: the nocturnal sleep and clinical and EEG evolution. Electroencephalogr Clin Neurophysiol 1977;43:312-316

6. Fonseca LC, Tedrus GMA. Pontas parietais evocadas ao EEG convulsões febris. Arq Neuropsiquiatr 1988;46:127-132.

7. Fonseca LC, Tedrus GMA, Odashima NS. Epilepsy syndromes in children with somatosensory evoked spikes. Epilepsia 1991:32(Suppl):88.

8. Dalla Bernardina B, Sgrò V, Caraballo R, et al. Sleep and benign partial epilepsies of childhood: EEG and evoked potentials study. Epilepsy Res 1991;2:83-96.

9. Zhu Y, Georgesco M, Cadilhac J. Normal latency value of early cortical somatosensory evoked potentials in children. Electroenceph Clin
Neurophysiol 1987;68:471-474.

10. Gilmore R. The use of somatosensory evoked potentials in infants and children J Child Neurol 1989;4:3-19.

11. Mutoh K, Hojo H, Mikawa H. Maturational study of short latency somatosensory evoked potentials after posterior tibial nerve stimulation in infants and children. Clin Neurophysiol 1989;20:91-102.

12. Chiappa K H. Evoked potentials in clinical medicine.2.Ed. New York: Raven Press. 1990.

13. Tassinari CA, De Marco P, Plasmati R, Pantieri R, Blanco M, Michelucci R. Extreme somatosensory evoked potentials (ESEPS) elicited by tapping of hands and feet in children: a somatosensory cerebral evoked potentials study. Neurophysiol Clin 1988;18:123-128.

14. Plasmatti R, Michelucci R, Forti A, et al. The neurophysiological features of benign partial epilepsy with rolandic spikes. Epilepsy Res 1992;6:45-48.

15. Plasmati R, Michelucci R, Savi F, et al. Giant somatosensory responses evoked by electrical and mechanical stimulation in idiopathic epilepsies of childhood. Neurology 1990;40:320.

16. Plasmati R, Borghi AM, Lucchi D, Rubboli E, Tassinari CA. Somatosensory evoked potentials in patients with benign epilepsy with rolandic spikes. Epilepsia 1996;37:88.

17. Manganotti $P$, Zanette G. Contribution of motor cortex in geration of evoked spikes in patients with benign rolandic epilepsy. Clin Neurophysiol 2000;111:964-974

18. Jasper HH. The ten-twenty electrode system of the International Federation. Electroencephalogr Clin Neurophysiol 1958;10:371-375.

19. Tedrus GMAS. Potencial evocado somato-sensitivo em crianças com epilepsias benignas parciais da infância e pontas evocadas por estímulos somato-sensitivos no eletrencefalograma. Tese doutorado, UNICAMP. Campinas 2000.

20. Calzolari S, Rozza L, DeMarco P. Median nerve middle-long latency somatosensory evoked potentials in children with tactile evoked spikes. J Clin Neurophysiol 1997;14:523-528.

21. Farnarier G, Regis H, Roger J. Potentials evoques somesthesiques et myoclonus d'action. Rev EEG Neurophysiol 1985;15:37-43.

22. Ferri R, Musumeci AS, Elia M, Del Gracco S, Scuderi C, Bergonzi P. BIT - mapped somatosensory evoked potentials in the fragile $X$ syndrome. Neurophysiol Clin 1994;24:413-426.

23. Schmitt B, Thun-Hohenstein L, Molinari L, Superti-Furga A, Boltshauser E. Somatosensosensory evoked potentials with high cortical amplitudes: clinical data in 31 children. Neuropediatrics 1994;25:78-84.

24. Mauguière F, Desmedt JE, Courjon J. Astereognosis and dissociated loss of frontal or parietal components of somatosensory evoked potentials in hemispheric lesions. Brain 1983;106:271-311.

25. Rothwell JC, Obeso JA, Marsden CD. On the significance of giant somatosensory evoked potentials in cortical myoclonus. J Neurol Neurosurg Psychiatr 1984;47:33-42.

26. Gehin PH, Huttin B, Brichet B, Weber M. Signification des potentiels evoques somesthesiques (PES) d'amplitude anormalement elevee. Rev EEG Neurophysiol 1985;15:155-161.

27. Seeger J, Jacobi G. Registration of somatosensory evoked potentials in children with different affections of the CNS: occurrence of giant early cortical potentials. Electroencephalogr Clin Neurophysiol 1991;78:26.

28. Furlong PL, Wimalarana S, Harding GFA. Augmented P22-N31 SEP component in a patient with a unilateral space occupying lesion. Electroenceph Clin Neurophyisiol 1993;88:72-77.

29. Shibasaki H, Yamashita Y, Neshige R, Tobimatsu S, Fukui R. Pathogenesis of giant somatosensory evoked potentials in progressive myoclonic epilepsy. Brain 1985,108:225-240.

30. Obeso JA, Rothwell JC, Marsden CD. The spectrum of cortical myoclonus. Brain 1985;108:193-224. 